\title{
NONDUMPED OSCILLATIONS OF ELECTRON BEAM IN ION BEAM UNDULATOR WITH ELECTRON AND ION BEAMS WITH DIFFERENT RADII
}

\author{
Yu.Ya. Golub*, \\ Moscow Radiotechnical Institute, 132 Warshavskoye shosse, 113519 Moscow, Russia,
}

\begin{abstract}
Ion beam undulator consisting of a high current relativistic electron beam propagating co-axially with a nonrelativistic ion beam in the drift tube. External radii of electron and ion beams are considered different from each other. In a staty-state situation the oscillations of electron and ion beams are characterized by lengths of standing waves $\mathrm{M}$ and $\mathrm{L}$ correspondingly. The regime with $\mathrm{M}<<\mathrm{L}$ is suitable. Moreover, when ion beam radius less than electron beam radius, beams oscillations are dumped. When $\mathrm{M}>>\mathrm{L}$ ion beam undulator is worked, when electron beam radius less than ion beam radius, and when ion beam radius less than electron beam radius. New effect is took place in this case [1]. The nonlinear electric and magnetic fields are took place outside ion beam when ion beam radius less than electron beam radius. However, beautiful nondumped oscillations of electron beam are occur in the case. Now, the cause of the dumping absence is investigated.
\end{abstract}

\section{BASIC EQUATIONS}

In last ten years the possibilities of using of socalled ion undulator for FEL are investigated [35]. A principle of the work consists in the excitation of transverse oscillations of beam's electron when an injection into a region with noncompensated ion charge takes place. However, at this moment a self static fields of intense relativistic electron beam (IREB) and ion's dynamics are not taken into account. But in long pulseand continuous regimes ion's dynamics must be taken into consideration. Moreover, in these situations it is necessary to

*yurigolub@mtu-net.ru carry out an energy of ion oscillations from a system and to change disturbed part of ion background onto nondisturbed one. This tasks may be solved by using of ion beam undulator. We investigate the ion - beam undulator which consists of a nonrelativistic ion beam propagating co-axially with a high-current relativistic electron beam. The characteristics of ion-beam undulator in case equal beams radius were investigated in [2]. However the working of ion-beam undulator in the case with Different Radii is not investigate in [2].

In this paper a combined dynamics of IREB and ion beam, injected co-axially along z-axis, in common case, in presence of an uniform longitudinal magnetic field $\mathrm{B}_{0}$, is considered. It is supposed that a stationary ( $\partial / \partial \mathrm{t}=0, \mathrm{t}$-time) azimutally uniform $(\partial / \partial \theta=0)$ state is settled in a system, which is characterized by nonuniform on radial $r$ and axial $\mathrm{z}$ coordinates distributions of the beam and collective electromagnetic fields. For it's description the Vlasov equations for electron and ion distribution functions $\mathrm{f}_{\mathrm{e}, \mathrm{i}}\left(\mathrm{z}, \mathrm{r}, \mathrm{v}_{\mathrm{x}}, \mathrm{v}_{\mathrm{y}}, \mathrm{v}_{\mathrm{z}}\right)$ are used with the equations for scalar potential and three components of vector one in long-wave $\left(\partial^{2} / \partial z^{2}<<\Delta_{\perp}\right)$, where $\Delta_{\perp}$ is transverse part of Laplace operator. A boundary conditions for components of potentials followed from system's axial symmetry, a presence of conducting tube with radius $\mathrm{R}$ and gauge condition $\operatorname{div} \boldsymbol{A}=0$. The Vlasov equations are solved by macroparticle's method, the equation for potentials - by the grid method. The mathematical problem is reduced to evolution one in z-direction by the change of independent variable $\mathrm{t}$ on $\mathrm{z}$ with using the relation $d / d t=\left(1 / v_{z}\right) d / d z$, where $v_{z}$-velocity of given macroparticle. 


\section{ION BEAM UNDULATOR WITH DIFFERENT BEAMS RADII}

In a staty-state situation the oscillations of electron and ion beams are characterized by lengths of standing waves $\mathrm{M}$ and $\mathrm{L}$ correspondingly. The investigations show that the case are $\mathrm{M} \sim \mathrm{L}$ not suited for using because any small perturbation in one of the beams leads to fast (on the distance of order) reaction of the other one, which, in it's turn, increases the reason, and so on. This situation is analogous to instability. The regime with $\mathrm{M}<<\mathrm{L}$ is more suitable. But for the case, when ion beam radius smaller than electron beam radius it is not useful. Because of dumping electron beam oscillations in nonuniform fields of the ion beam. The useful regime is realized when $\mathrm{M}>>\mathrm{L}$ in the case different beams radius. In this case ion beams oscillations follows electron beam oscillations.

On Fig. 1 the dependencies of rms beams radii, normalized by $\mathrm{R}$, of electron and ion (proton in the case) beams on longitudinal coordinate $\xi$ $=\mathrm{z} / \mathrm{R}$ are shown. They are obtained in the simulation with electron current $0.5 \mathrm{kA}$, electron relativistic factor $=3$, longitudinal magnetic field $B=1 \mathrm{kG}$, ion beam density $\mathrm{ni}=\mathrm{f} * \mathrm{ne}, \mathrm{f}=2 / 9$, initial ion $\mathrm{z}$-velocities $=0.01 *_{\mathrm{c}}$ (c - speed of light), external electron beam radius $0.6^{*} \mathrm{R}$, external ion beam radius $0.4^{*} \mathrm{R}$.

Beautiful nondumped electron beam oscillations are shown. Such electron beam oscillations are useful in FEL with ion beam undulator. In this case ion beam nonlinear fields don't distort electron beam oscillations. Ion beam osculates with both wavelengths $\mathrm{M}$ and $\mathrm{L}$. The ion beam oscillations amplitude with wavelength $\mathrm{M}$ is large then with wavelength $\mathrm{L}$. Thus the ion beam is followed the electron beam oscillations.

Ion beam undulator beautiful work with electron and ion beams with different radii only in this case, when $\mathrm{M}>>\mathrm{L}$.

Cause of nondumped electron beam oscillations is strong difference between lengths of standing waves of electron and ion beams. The change of radius of ion beam changes area,

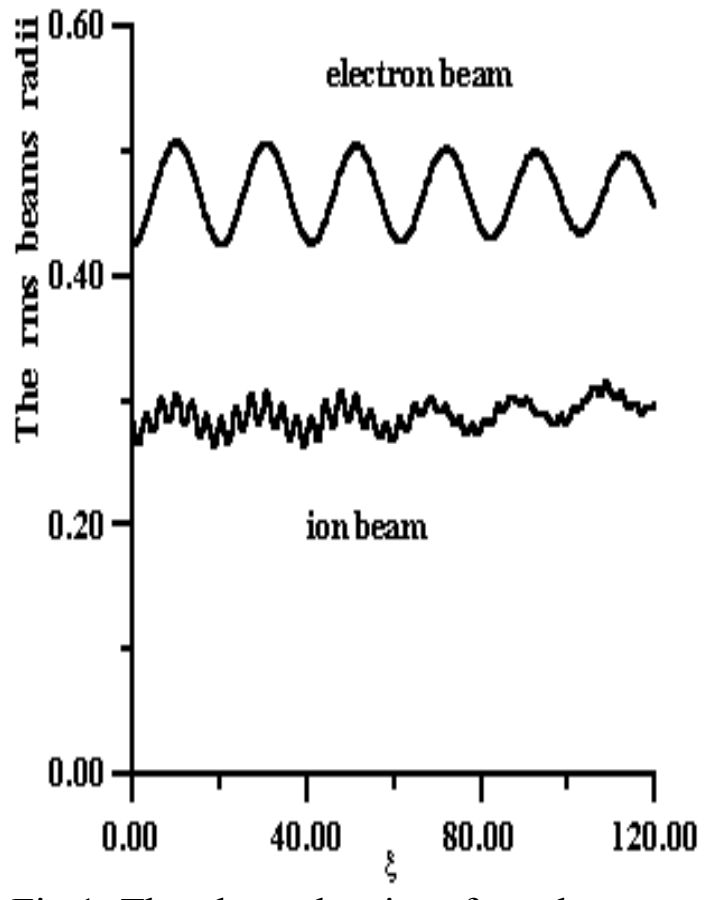

Fig.1: The dependencies of rms beams radii, normalized by $\mathrm{R}$, of electron and ion (proton in the case) beams on longitudinal coordinate $\xi$ $=\mathrm{z} / \mathrm{R}$.

where the fields are non-linear. So radial nonlinear fields depend from z. It appears if to average radial nonlinear fields on $\mathrm{z}$ that they will be linear on lengths about length of oscillations of electrons. Therefore the electron beam propagate in linear fields on the average.

\section{REFERENCES}

[1]. Yu.Ya. Golub, EPAC Proceedings, 1298 (2000)

[2]. Yu.Ya. Golub, N.E.Rozanov, Nuclear Instruments and Methods in Physics Research, A358 (1995) 479

[3]. R.A. Bosch and R.M. Gildenbach, Phys.Fl., 31 (1988) 3127.

[4]. D.H. Whittum and A.M. Sessler, Phys.Rev.Lett. 64 (1990) 2511.

[5]. R.K. Chen, T.C. Katsouleas, and J.M. Dawson, JEEE Transac. on Pl.Sci. 18 (1990) 837. 